\title{
"Design of pulmonary rehabilitation programmes during acute exacerbations of COPD: a systematic review and network meta-analysis." Ana Machado, Pedro Matos Silva, Vera Afreixo, Cátia Caneiras, Chris Burtin and Alda Marques. Eur Respir Rev 2020; 29: 200039.
}

Copyright (T) The authors 2021

This version is distributed under the terms of the Creative Commons Attribution Non-Commercial Licence 4.0. For commercial reproduction rights and permissions contact permissions@ersnet.org

\section{๑@®®}

Unfortunately, in this article in the December 2020 issue of the European Respiratory Review figure 2b was presented incorrectly. The WMD data for "Exercise", "Breathing techniques" and "Education and psychosocial support and breathing techniques” were incorrectly present with minus symbols.

The correct figure is published below.

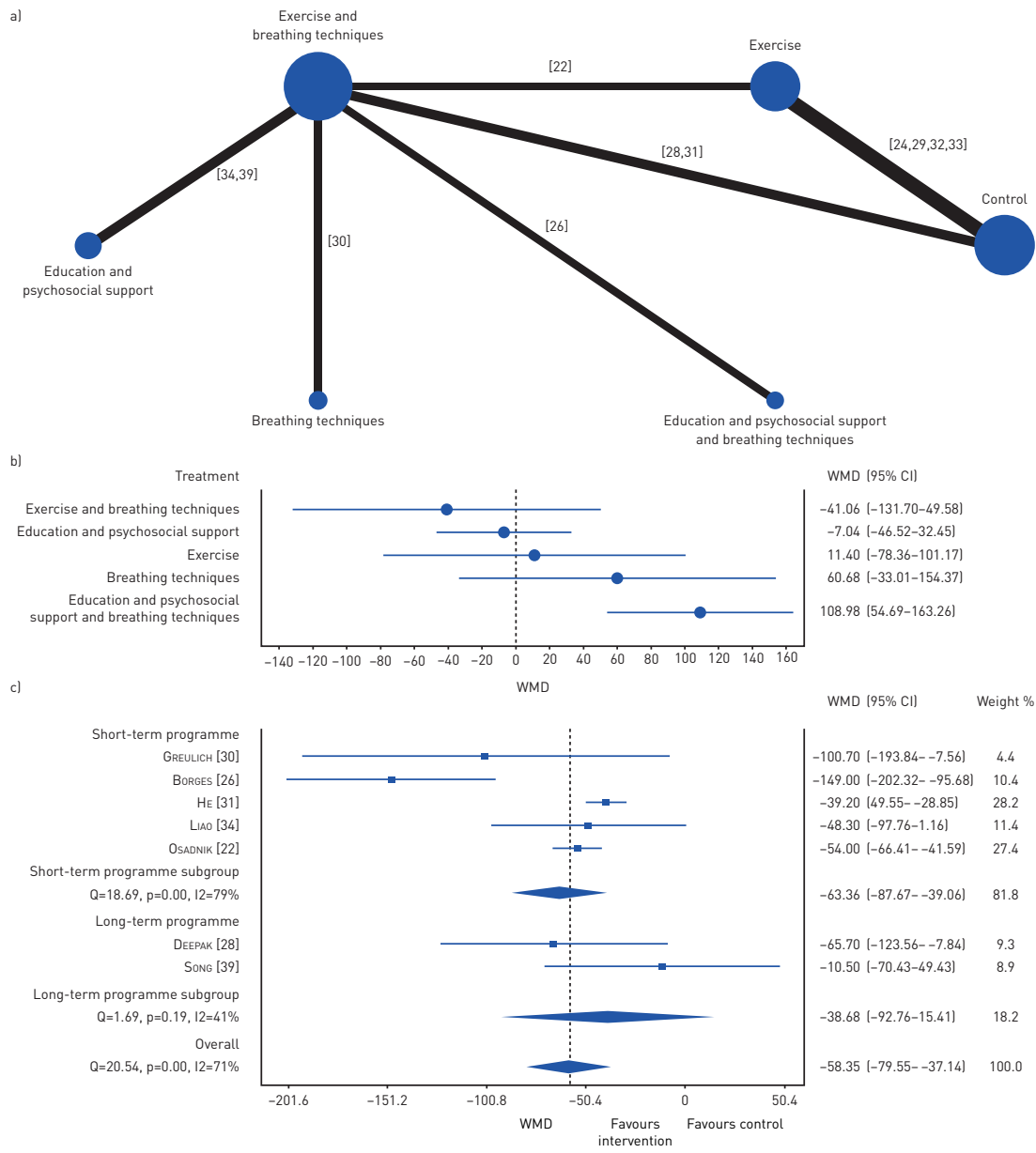

FIGURE 2 Effects of the different interventions on exercise capacity measured with 6-min walk test. a) Network plot of treatments with nodes representing the number of interventions being compared and edges representing the available direct comparisons (comparisons evaluated in at least one study) between pairs of interventions. b) Forest plot of network meta-analysis results (all interventions versus standard of care). c) Forest plot of comparison of short-term programmes versus long-term programmes using a combination of exercise with breathing techniques. WMD: weighted mean difference. 